\title{
SHORT GEODESIC LOOPS ON COMPLETE RIEMANNIAN MANIFOLDS WITH A FINITE VOLUME
}

\author{
REGINA ROTMAN
}

\begin{abstract}
In this paper we will show that on any complete noncompact Riemannian manifold with a finite volume there exist uncountably many geodesic loops of arbitrarily small length.
\end{abstract}

\section{INTRODUCTION}

In the paper we will prove the following theorem:

Theorem 0.1. Let $M^{n}$ be a complete noncompact Riemannian manifold of a finite volume $V$. Then, given a point $p \in M^{n}$ and $\varepsilon>0$, there exists a set $A \subset(0, \infty)$ of measure $\frac{V}{f(\varepsilon)}$, where $f(\varepsilon)=\left(\frac{\varepsilon}{12 \cdot 108^{n-1} n !}\right)^{n-1}$, such that for every $t$ not in $A \cup\left(0, \frac{\varepsilon}{2}\right)$ there exists a geodesic loop on $M^{n}$ of length $\leq \varepsilon$ based at the distance $t$ from the point $p$.

Remarks. (1) Theorem 0.1 immediately implies that the set of distinct geodesic loops of length $\leq \varepsilon$ on $M^{n}$ is uncountable.

(2) As it will be seen from the proof, the theorem also applies to closed Riemannian manifolds and $\varepsilon>0$ providing that $r_{p}=\max _{q \in M^{n}} \operatorname{dist}(p, q)>\frac{V}{f(\varepsilon)}+\varepsilon$ with the conclusion valid for the values of $t \in\left(\frac{\varepsilon}{2}, r_{p}-\frac{\varepsilon}{2}\right)$ in the complement of $A$.

Note that the existence of arbitrarily short geodesic loops on a complete noncompact Riemannian manifold of a finite volume also easily follows from the theorem below proven by S. Sabourau (see [S2]):

Theorem 0.2 ([S2]). Let $M^{n}$ be a complete Riemannian manifold of dimension $n$. Then there exists $C(n)>0$, such that the volume of any ball $B\left(x_{0}, r\right)$ of radius $r \leq \frac{\operatorname{sgl}\left(M^{n}\right)}{2}$, where $\operatorname{sgl}\left(M^{n}\right)$ is the length of a shortest geodesic loop on $M^{n}$, is at least $C(n) r^{n}$.

It is clear from Sabourau's argument that one can, alternatively, define $\operatorname{sgl}\left(M^{n}\right)$ as the the infimum of lengths of geodesic loops on $M^{n}$ in order to cover the situation when there is no shortest geodesic loop on $M^{n}$. In this case the same lower bound for the volume of metric balls will still hold. This will immediately imply that if $M^{n}$ is a complete and noncompact Riemannian manifold with a finite volume, then $\operatorname{sgl}\left(M^{n}\right)=0$, as otherwise, one would have an infinite set of disjoint metric balls with volumes uniformly bounded from below. Their combined volume will be infinite contradicting the fact that $M^{n}$ has a finite volume.

Received by the editors April 28, 2011.

2010 Mathematics Subject Classification. Primary 53C22, 53C23. 
Yet, it is not clear how one can adapt Theorem 0.2 from S2 to derive more information about short geodesic loops. In particular, the existence of uncountably many loops of length at most $\varepsilon$ is not guaranteed by his method.

In this paper we also prove the following result:

Theorem 0.3. Let $M^{n}$ be a complete noncompact Riemannian manifold of a finite volume $V$. Then given a point $p \in M^{n}$ there exists $T>0$, such that for all $t>T$ there exists a geodesic loop of length at most $\varepsilon$ at the distance $t$ from $p$.

Note that, if desired, one can combine the statements of Theorems 0.1 and 0.3 by demanding that the set $A$ in the text of Theorem 0.1 is bounded.

In view of Theorem 0.3 one can ask if given an $\varepsilon>0$ there exists $T>0$ such that for every $t>T$ and every point $q \in M^{n}$ at the distance $t>T$ from $p$ there exists a geodesic loop of length at most $\varepsilon$ based at $q$. The answer to this question is negative. To see this consider a complete noncompact manifold of a finite volume and attach to it an infinite sequence of long cylindrical "fingers" of exponentially decreasing radii that are capped by hemispheres $H_{i}$. Assume that the sequence of the distances from the bases of these fingers to $p$ are unbounded. It is clear that there are no short geodesic loops based at the centers of $H_{i}$.

These theorems provide an answer to one of many questions about the relationship between the volume of a complete noncompact Riemannian manifold and lengths of various stationary objects.

Previously, questions of a similar nature were investigated by C. B. Croke, who has established a volume upper bound for the length of a shortest periodic geodesic on a surface of a finite volume (see [C]) and by S. Sabourau, who has indicated how to bound the length of a shortest geodesic loop on a complete Riemannian manifold by its volume (see [S2]) as well as by M. Gromov (see [G]), who obtained some estimates for 1-systoles in the case of manifolds that are essential relative to infinity and manifolds that have essential ends.

Note that it is not known whether on any complete Riemannian manifold of finite volume of dimension greater than 2 there exists a periodic geodesic (though it was shown by V. Bangert and G. Thorbergsson that there exist infinitely many geodesics on a complete surface of a finite volume (see $[\mathrm{B}]$ and $[\mathrm{T}])$ ).

In the case of a closed Riemannian manifold $M^{n}$, there are numerous results that connect the size (i.e. the length or the area) of various stationary objects, such as geodesic loops, minimal geodesic cycles and nets, or minimal surfaces or submanifolds to the size of a manifold as measured either by its volume or the diameter (see, for instance, [B1], [NR2], [NR3, [NR4, R2, [R3], [R4], S2]).

Presently there are no general curvature-free upper bounds of this nature for the length of a shortest periodic geodesic on a simply connected manifold, except in dimension 2 (see [C], [M], NR1], R1], S1]), though many results for manifolds with nontrivial fundamental group are known (see [BZ], CK] for surveys of these results). The most notable is the result of $\mathrm{M}$. Gromov, which gives a volume estimate for the length of a shortest periodic geodesic on closed Riemannian manifolds that are essential (see G]).

Our proof will make use of the following definition and result by M. Gromov. (See [G] as well as the recent paper by S. Wenger [W]. Wenger provides a short proof of the filling volume versus volume inequality, which is at the core of Gromov's original proof. Wenger's paper also implies some improvements of the original result, in particular, an improvement of the dimensional constant.) We will also 
use some ideas from Gromov's paper [G] and our approach of constructing "fillings" of cycles in the absence of short geodesic loops used in [R3].

Definition 0.4 (Filling Radius [G]). Let $M^{n}$ be an $n$-dimensional Riemannian manifold in an arbitrary metric space $X$. Then the filling radius $\operatorname{Fill} \operatorname{Rad}\left(M^{n} \subset X\right)$ is the infimum of $\varepsilon>0$, such that $M^{n}$ bounds in the $\varepsilon$-neighborhood $N_{\varepsilon}\left(M^{n}\right)$, that is, $i_{*}\left(H_{n}\left(Z_{n}\right)\right)=\{0\}$, where $i_{*}$ is induced by the inclusion $i: M^{n} \longrightarrow N_{\varepsilon}\left(M^{n}\right)$ and where $H_{n}\left(M^{n}\right)$ is taken with coefficients in $\mathbf{Z}$ when $M^{n}$ is orientable and with coefficients in $\mathbf{Z}_{2}$ when $M^{n}$ is nonorientable. The filling radius of an abstract Riemannian manifold is then defined to be $\operatorname{Fill} \operatorname{Rad}\left(M^{n} \subset L^{\infty}\left(M^{n}\right)\right)$, where the Kuratowski embedding of $M^{n}$ into $L^{\infty}\left(M^{n}\right)$ is a map that to each point $p$ of $M^{n}$ assigns a distance function $p \longrightarrow f_{p}=d(p, q)$. Equivalently, Fill $\operatorname{Rad} M^{n}$ can be defined as the infimum of Fill $\operatorname{Rad}\left(M^{n} \subset X\right)$ over all metric spaces $X$ and isometric embeddings of $M^{n}$ into $X$.

Theorem $0.5(\mathrm{G})$. Let $M^{n}$ be an $n$-dimensional manifold. Then Fill $\operatorname{Rad} M^{n} \leq$ $k(n) \operatorname{vol}\left(M^{n}\right)^{\frac{1}{n}}$, where $k(n)$ is an explicit function of the dimension of a manifold.

Gromov's dimensional constant $k(n)=(n+1) n^{n} \sqrt{(n+1) !}$ can be improved to $\tilde{k}(n)=27^{n}(n+1)$ ! by combining the result by Wenger in $\mathrm{W}$ with the inequality (2.6) in G].

Note that L. Guth has recently proved an important improvement of the above result by showing that a complete Riemannian manifold with the filling radius $R$ contains a ball of radius $R$ of volume bounded from below by $c(n) R^{n}$ (see Gt1]).

\section{Three SIMPLE LEMMAS}

We will begin the proof of the main results with the following three lemmas.

Lemma 1.1. Let $M^{n}$ be a complete noncompact Riemannian manifold of a finite volume $V, p \in M^{n}$. Let $\sigma(t)$ be a geodesic ray, starting at a point $p$. Then given $\tilde{\varepsilon}>$ 0 , there exists a set $A=A(\tilde{\varepsilon}) \subset(0, \infty)$ of measure at most $\frac{16 V}{\tilde{\varepsilon}}$, such that for every $t^{*}$ in $A^{c}$ (the complement of $A$ in $(0, \infty)$ ), and for every $0<\delta<\min \left\{1, \frac{\tilde{\varepsilon}}{2}\right\}$ there exists an $(n-1)$-dimensional submanifold $Z_{\tilde{\varepsilon}}^{\delta}$ of $M^{n}$ with the following properties:

(1) $\operatorname{vol}_{n-1}\left(Z_{\tilde{\varepsilon}}^{\delta}\right)<\tilde{\varepsilon}$;

(2) $Z_{\tilde{\varepsilon}}^{\delta}$ does not bound in $M^{n} \backslash\{p\}$;

(3) the distance between $Z_{\tilde{\varepsilon}}^{\delta}$ and the geodesic sphere $\tilde{S}_{t^{*}}(p)=\left\{x \in M^{n} \mid \operatorname{dist}(x, p)=\right.$ $\left.t^{*}\right\}$ is at most $\delta$.

Proof. Let $\tilde{S}_{t}(p)$ be a family of geodesic spheres centered at the point $p$ of radius $t \in(0, \infty)$. By the coarea formula $\int_{0}^{\infty} \operatorname{vol}_{n-1}\left(\tilde{S}_{t}(p)\right) d t=V$. Therefore, there exists a set $A=A(\tilde{\varepsilon})$ of measure at most $\frac{16 V}{\tilde{\tilde{\varepsilon}}}$ such that for any $t^{*} \in A^{c}$ the $(n-1)$-dimensional Hausdorff measure $\operatorname{vol}_{n-1}\left(\tilde{S}_{t^{*}}\right)$ is at most $\frac{\tilde{\varepsilon}}{16}$. Moreover, by the continuity of volume, for any $t^{*}$ there exists a small neighborhood $\left(t^{*}-\tau, t^{*}+\tau\right)$ of $t^{*}$ such that for any $t \in\left(t^{*}-\tau, t^{*}+\tau\right), \operatorname{vol}_{n-1}\left(\tilde{S}_{t}(p)\right)<\frac{\tilde{\varepsilon}}{8}$. Thus, $\int_{t^{*}-\tau}^{t^{*}+\tau} \tilde{S}_{t}(p) d t<\frac{\tilde{\varepsilon} \tau}{4}$.

Let $0<\delta<\min \left\{1, \frac{\tilde{\varepsilon}}{2}\right\}$ be given.

Let $\varrho_{\sigma}: M^{n} \longrightarrow \mathbf{R}, \sigma<\delta$ be a function that is smooth on $M^{n} \backslash\{p\}$ and that approximates a distance function $\varrho_{p}$ from the point $p$ in the following way: (1) $\varrho_{\sigma}=\varrho_{p}$ on a geodesic ball centered at $p$ of radius smaller than the injectivity radius of $M^{n}$ at $p ;(2)\left|\varrho_{p}-\varrho_{\sigma}\right| \leq \sigma$; and $(3)\left|\operatorname{grad} \varrho_{\sigma}\right| \leq 1+\sigma$. The details of constructing such a function can be found in M. P. Gaffney's work Ga]. 
Let us now consider the level sets $S_{t}(p)$ of $\varrho_{\sigma}$. By Sard's theorem, the level sets will be $(n-1)$-dimensional submanifolds of $M^{n}$ for almost all $t$. For some small values of $t \in \mathbf{R}$, they will be geodesic spheres, because $\varrho_{\sigma}$ agrees with the distance function in some neighborhood of $p$. Let $\tilde{S}_{r}(p)$ be a geodesic sphere centered at $p$ with radius $r$ smaller than the injectivity radius at the point $p$. Then $\tilde{S}_{r}(p)$ is homeomorphic to $S^{n-1}$.

By the virtue of the Mayer-Vietoris exact sequence, it follows that $\tilde{S}_{r}(p)$ does not bound in $M^{n} \backslash\{p\}$. Otherwise, $H_{n}\left(M^{n}\right) \neq\{0\}$, which would contradict the assumption that $M^{n}$ is not compact. Now consider $Q \subset(0, \infty)$ of all values $t$ for which $S_{t}(p)$ is an $(n-1)$-dimensional submanifold of $M^{n}$. It is clear that $S_{t_{1}}$ and $S_{t_{2}}$ are homologous in $M^{n}-p$ for all $t_{1}, t_{2} \in Q$. Therefore, $S_{t}(p)$ does not bound in $M^{n}-p$ for all $t \in Q$, and, thus, for almost all $t \in(0, \infty)$.

Without any loss of generality we can assume that $\tau<\delta$. Let $\sigma=\frac{\tau}{2}$. Consider $\int_{t^{*}-\frac{\tau}{2}}^{t^{*}+\frac{\tau}{2}} \operatorname{vol}_{n-1}\left(S_{t}(p)\right) d t$, which, by coarea formula is at most $\frac{\tilde{\varepsilon} \tau(1+\sigma)}{4} \leq \frac{\tilde{\varepsilon} \tau}{2}$. Thus, there exists a set $B \subset\left(t^{*}-\frac{\tau}{2}, t^{*}+\frac{\tau}{2}\right)$ of measure at least $\frac{\tau}{3}$, such that $\operatorname{vol}_{n-1}\left(S_{t}(p)\right)<$ $\tilde{\varepsilon}$ for every $t \in B$.

Furthermore, when $t \in B$ the distance between $\tilde{S}_{t^{*}}(p)$ and $S_{t}(p)$ is at most $\delta$. Now let us select $t \in B$ so that this $S_{t}(p)$ is a submanifold, and let $Z_{\tilde{\varepsilon}}^{\delta}=S_{t}(p)$. We have shown that $Z_{\tilde{\varepsilon}}^{\delta}$ has the desired properties.

The following two lemmas were used in [R3]. We will present them here for the sake of completeness.

The first is a Morse-theoretic type lemma asserting that the space of loops based at a fixed point $q$ of length smaller than the length of a minimal geodesic loop at $q$ is contractible.

Lemma 1.2. Let $M^{n}$ be a complete Riemannian manifold. Let $q \in M^{n}$. Suppose that the length of a shortest geodesic loop $l_{q}\left(M^{n}\right)$ based at $q$ is greater than $L$. Then, given any piecewise differentiable loop $\gamma:[0,1] \longrightarrow M^{n}$ of length $\leq L$ such that $\gamma(0)=\gamma(1)=q$, there exists a length decreasing path homotopy connecting this curve with $q$ that depends continuously on the initial loop $\gamma$.

Proof. There is a standard explicit length shortening deformation of the space of loops based at $q$ of length $\leq L$ to the constant loop via the Birkhoff Curve Shortening Process (BCSP). (See [C] for a detailed description of the BCSP for closed curves. The only difference between the BCSP for closed curves and the BCSP for loops is that one fixes a basepoint during the homotopies in the latter case.)

The third lemma can be viewed as an effective version of an elementary assertion that two curves $\gamma_{1}, \gamma_{2}$ connecting points $q_{1}, q_{2}$ are path homotopic if and only if the loop $\gamma_{2} *-\gamma_{1}$ is path homotopic to a point. Lemma 1.3 is analogous to a similar statement in $\mathrm{C}$, namely Lemma 3.1.

Lemma 1.3. Let $\gamma_{1}, \gamma_{2}$ be two curves with $\gamma_{1}(0)=\gamma_{2}(0)=q_{1}$ and $\gamma_{1}(1)=\gamma_{2}(1)=$ $q_{2}$ on a complete Riemannian manifold $M^{n}$ of length $l_{1}, l_{2}$, respectively.

Let $\gamma_{2} *-\gamma_{1}$ be the product of $\gamma_{2}$ and $-\gamma_{1}$ based at $q_{1}$. If this curve is contractible to $q_{1}$ as a loop along the curves of length $\leq l_{1}+l_{2}$, then there is a path homotopy (i.e. a homotopy that fixes the endpoints) $h_{\tau}(t), \tau \in[0,1]$, such that $h_{0}(t)=\gamma_{1}(t), h_{1}(t)=\gamma_{2}(t)$, and the length of curves during this homotopy is bounded above by $2 l_{1}+l_{2}$. Alternatively there exists a path homotopy with the same properties, such that the length of curves in it is bounded by $l_{1}+2 l_{2}$. Moreover, 

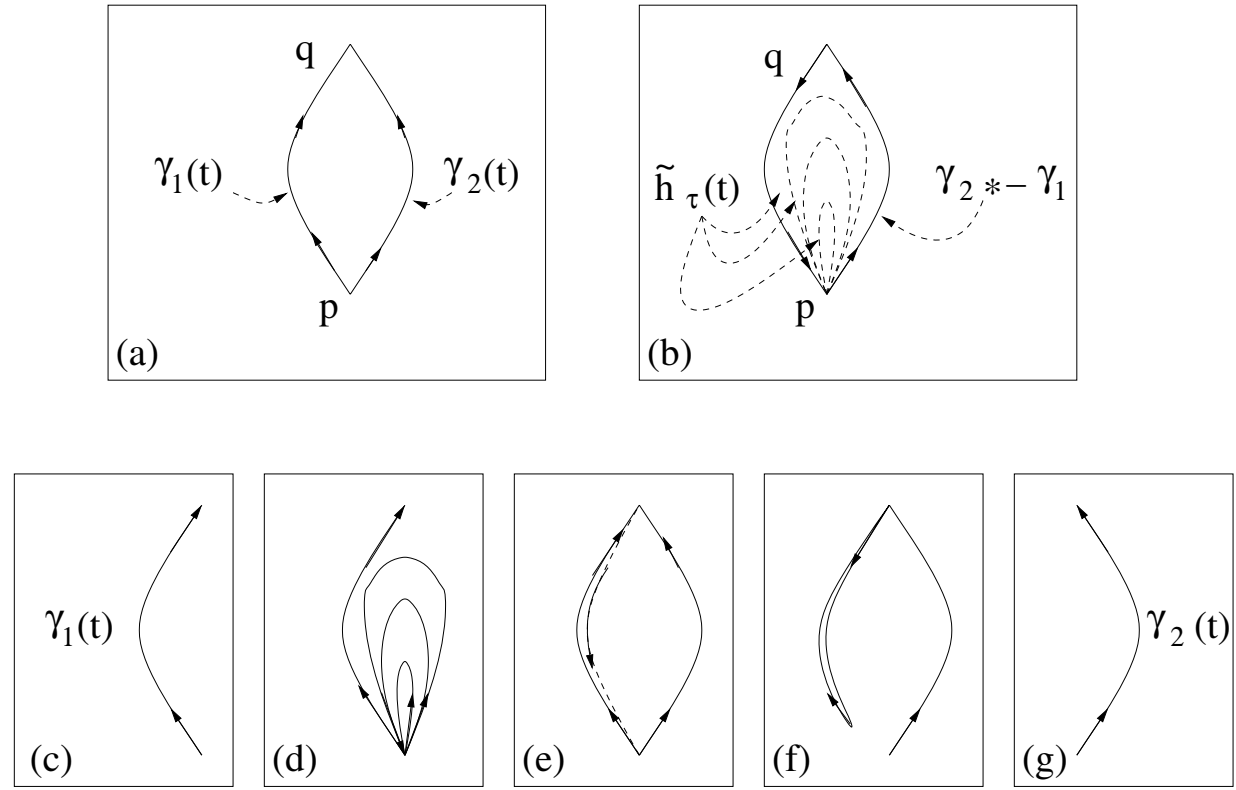

Figure 1. Illustration of the proof of Lemma 1.3

when $M^{n}$ has no geodesic loops of length $\leq l_{1}+l_{2}$, this path homotopy can be made to continuously depend on the digon formed by $\gamma_{1}$ and $\gamma_{2}$; see Lemma 1.2 .

Proof. Let $\tilde{h}_{\tau}(t)$ be a homotopy that connects $\gamma_{2} *-\gamma_{1}$ with a point $p$ (see Figure 1 (a) and (b)). Then let us consider the homotopy $\gamma_{1} \sim \tilde{h}_{1-\tau} * \gamma_{1} \sim \gamma_{2} *-\gamma_{1} * \gamma_{1} \sim \gamma_{2}$ (see Figure 1 (a)-(g)). The length of curves during this homotopy is $\leq 2 l_{1}+l_{2}$.

Note that, assuming there are no geodesic loops of length $\leq l_{1}+l_{2}$, one can contract $\gamma_{2} *-\gamma_{1}$ via the BCSP, which continuously depends on the initial curve (see Lemma 1.2). Thus, the path homotopy between $\gamma_{1}(t)$ and $\gamma_{2}(t)$ will also continuously depend on the initial digon.

Also, one can reverse the role of $\gamma_{1}$ and $\gamma_{2}$ and construct a path homotopy between $\gamma_{2}$ and $\gamma_{1}$ passing through curves of length $l_{1}+2 l_{2}$. Then we reverse the direction of this path homotopy obtaining a path homotopy from $l_{1}$ to $l_{2}$ with the required properties.

\section{Proof of Theorems 0.1 and 0.3}

In the following definition (Definition 3.1 in [R3]), we let $\sigma^{m+1}$ denote the standard $(m+1)$-dimensional simplex and $C(X, Y)$ denote the space of continuous maps from $X$ into $Y$.

Definition 2.1. Given $l>0$ and a positive integer $m$, let $K_{m, l}$ be a space of piecewise smooth maps of the complete graph with $(m+2)$ vertices into $M^{n}$, such that each edge is mapped into a curve of length $\leq l$. We define an $N$-filling of $K_{*, l}$ as a collection of continuous maps $\phi_{m}: K_{m, l} \longrightarrow C\left(\sigma^{m+1}, M^{n}\right), m=1,2, \ldots, N$, satisfying the following properties:

(1) For every $k \in K_{m, l}$, the restriction of $\phi_{m}(k)$ to the 1-skeleton of $\sigma^{m+1}$ coincides with $k$, that is, each $\phi_{m}(k)$ extends $k$. 
(2) For every $k \in K_{m, l},(m \leq N)$, the restriction of $\phi_{m}(k)$ to an $m$-dimensional face of $\sigma^{m+1}$ coincides with $\phi_{m-1}$ evaluated on the element of $K_{m-1, l}$ obtained from $k$ by restricting $k$ to the set of all 1-dimensional simplices in the 1-skeleton of this face of $\sigma^{m}$.

Here is an informal description of the above definition: We are "filling" graphs with "short" edges (i.e. of length $\leq l$ ) that correspond to the immersed 1-skeleton of a simplex of dimension $m+1$ by discs of dimension $m+1$, so that the map of the disc extends the map from the 1-skeleton. Moreover, this extension is done in a coherent way; that is, if we consider the restriction of this map to a face of the simplex, it will be a "filling" of the 1-skeleton of the face. In particular, that means that each $N$-filling agrees with its subfillings and depends continuously on its 1-skeleton.

Lemma 2.2. Suppose that the length of a shortest geodesic loop on $M^{n}$ is greater than $3 \cdot 4^{n-1} l$. Then there exists an $n$-filling of $K_{*, l}$. Moreover, if $k \in K_{m, l}$ $(m \leq n)$, the disc that fills $k$ will lie in the $6 \cdot 4^{n-2} l$-neighborhood of the set of vertices of $k$; that is, the maximal distance between points of the disc and the set of vertices of $k$ is at most $6 \cdot 4^{n-2} l$.

Proof. We will prove the existence of the $i$-fillings of $K_{*, l}$ for every $i \leq n$. The proof will be by induction with respect to $i$. The base step corresponds to $i=1$. Let $k_{1} \in K_{1, l}$. By Definition 2.1 it is an immersion of a full graph that consists of three vertices and three edges. Let $v_{0}, v_{1}, v_{2}$ be the vertices of this immersed graph. The three edges form a loop based at $v_{0}$. Since we have assumed that there are no "short" geodesic loops (and, in particular, no geodesic loops of length $\leq 3 l$ ), this loop is contractible to $v_{0}$ via shorter loops based at $v_{0}$. This homotopy generates a disc that "fills" $k^{1}$.

At each subsequent step, to construct $\phi_{j}$ we consider its restriction to $\partial \sigma^{j+1}$. This restriction is uniquely determined by the definition of $N$-fillings and, if $i>1$, by the previous steps of the induction. That is, the previous step of the induction results in a filling of elements of $K_{j-1, l}$ obtained from elements of $K_{j, l}$ by deleting a vertex. Consider $k \in K_{j, l}$. Then the fillings of $j+2$ elements of $K_{j-1, l}$ that are obtained from $k$ by deleting a vertex are discs of dimension $j$ provided by the previous step of the construction. They together form a $j$-dimensional sphere, which, according to our definition, is a restriction of $\phi_{j}$ to $\partial \sigma^{j+1}$. The required disc is then generated by a homotopy that contracts this sphere to a point. To construct this homotopy, we begin by constructing a 1-parameter family $k_{t}$ of immersed graphs connecting $k=k_{0}$ with a complete graph $k_{1}$ with $(j+2)$ vertices immersed in $M^{n}$ such that all of its edges are mapped to some paths in a tree. This path $k_{t}$ should continuously depend on the initial graph $k$. Next, we construct a 1parameter family of spheres $S_{t}^{j}$ by filling all $k_{t}$ 's. This results in a homotopy between the sphere $\phi_{j}\left(\partial \sigma^{j+1}\right)$ and the degenerate sphere $S_{1}^{j}$ that lives in a tree and is, therefore, contractible. (In order to contract this degenerate sphere, we contract $k_{1}$ over itself and fill it by the $n$-sphere at each moment of the homotopy.) For every $t, k_{t}$ is constructed by several applications of an operation of a collapsing of a triangle: Let $k_{a}, k_{b}, k_{c}$ be any three edges of $k$ forming a triangle. As there are no geodesic loops of length $\leq$ length $k_{a}+$ length $k_{b}+$ length $k_{c}$ in $M^{n}$, we can apply Lemma 1.3 to construct a path homotopy between $k_{a}$ and $k_{b} * k_{c}$. This homotopy passes through paths of length $\leq 2 \operatorname{length}\left(k_{a}\right)+\operatorname{length}\left(k_{b}\right)+\operatorname{length}\left(k_{c}\right) \leq 4 l$. This 


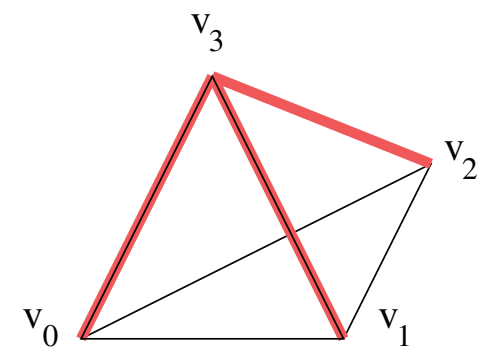

Figure 2. Collapsing triangles.

homotopy induces a homotopy of triangles $\left(k_{a}\right)_{t}, k_{b}, k_{c}, t \in[0,1]$ that we call a collapsing of the triangle $k_{a}, k_{b}, k_{c}$. At the end of this homotopy $k_{a}$ is being replaced by another edge that goes along $k_{b} * k_{c}$, and the considered triangle becomes thin. Note that when one is given a triangle with the sides $k_{a}, k_{b}, k_{c}$ there is a freedom of what side is being deformed and which vertex is used as a basepoint for contracting a loop. To avoid ambiguity, we can assume that the side $k_{a}$ that is being deformed is the one that connects vertices with the smallest indices, and the loops are always being contracted to a vertex with the smallest index.

After collapsing finitely many triangles, we can obtain an element of $K_{j, 4 l}$, where all edges run along the tree-shaped union $k_{1}$ of edges of $k$ adjacent to one vertex of $k$, let us say the vertex with the highest number (see Figure 2, which illustrates that the edge $\left[v_{0}, v_{1}\right]$ is being collapsed to the path $\left[v_{0}, v_{3}, v_{1}\right]$, the edge $\left[v_{1}, v_{2}\right]$ is being collapsed to $\left[v_{1}, v_{3}, v_{2}\right]$, and the edge $\left[v_{0}, v_{2}\right]$ is being collapsed to $\left.\left[v_{0}, v_{3}, v_{2}\right]\right)$.

Now we can continue the homotopy of complete graphs by contracting all edges of $k_{1}$ to a point (to $v_{3}$ on Figure 2) along the tree by a length nonincreasing homotopy.

The resulting graphs are filled by $j$-spheres using the induction assumption on $K_{m, 4 l}$, since the length of edges that result in the process of collapsing of triangles is bounded above by $4 l$.

Let $k \in K_{m, l}$. Then $k$ is (a map of) the complete graph with $m+2$ vertices $v_{0}, v_{1}, \ldots, v_{m+1}$. Let $k_{t_{1}}$ denote a 1 -parameter family of graphs obtained from $k$ by collapsing triangles. We define $k_{t_{1}}^{i_{1}}$ to be a subgraph of $k_{t_{1}}$ obtained from it by removing a vertex $v_{i_{1}}$. In general, let $k_{t_{1}, \ldots, t_{j}}^{i_{1}, \ldots, i_{j-1}}$ be a family of complete graphs with $m+3-j$ vertices obtained from $k_{t_{1}, \ldots, t_{j-1}}^{i_{1}, \ldots, i_{j-1}}$ by collapsing triangles, and let $k_{t_{1}, \ldots, t_{j}}^{i_{1}, \ldots, i_{j}}$ be the complete graph with $m+2-j$ vertices obtained from $k_{t_{1}, \ldots, t_{j}}^{i_{1}, \ldots, i_{j-1}}$ by removing a vertex $v_{j}$. Let $a(j)$ be the maximal possible length of an edge of $k_{t_{1}, \ldots, t_{j}}^{i_{1}, \ldots, i_{j}}$. Note that $a(1) \leq 4 l$ and that $a(j+1) \leq 4 a(j)$. Thus, $a(m-1) \leq 4^{m-1} l$. So, the length of loops that one contracts in the recursive process described above is at most $3 \times 4^{n-1} l$.

Note also, that as all the homotopies are constructed by contracting loops to one of the vertices of $k$, the maximal distance from the points of the resulting disc to one of the vertices is at most half the maximal length of such loops.

Remark 1. This lemma is analogous to the filling technique invented by S. Sabourau in [S2] to show that in the absence of "short" geodesic loops one can contract "small" spheres, but is different from it. As far as we are aware, [S2] is the first paper that introduced such a filling technique. 
Remark 2. Assume that we are applying the above proof to fill an individual $k \in$ $K_{m, l}$. In the course of the construction, we need to contract the loops that are based at the vertices of $k$ by path homotopies that pass through loops that are short. Moreover, two vertices with the highest indices are never used. Therefore, to fill $k$ only the absence of "short" geodesic loops based at all vertices of $k$ but the two with the highest indices is required.

Here is the informal description of the above proof when $m=2$. We would like to show that in the case when the length of a shortest geodesic loop is $>12 l$ we can fill $K_{2, l}$. Let us recall that $K_{2, l}$ is the space of an immersed 1-skeleta of simplices of dimension 3 , such that the length of each edge does not exceed $l$. We would like to extend each of the immersions to a 3 -simplex, so that these extensions are continuous with respect to the original graph and so that they are coherent. The last requirement means that if we consider a restriction of the immersion to a subcomplex, which is a 1-skeleton of a 2 -face, it will agree with the earlier extension. Thus, the procedure is inductive, and we will begin by filling $K_{1,4 l}$. In this case, if $k \in K_{1,4 l}$, then its total length is at most $12 l$. However, since the length of a shortest geodesic loop is greater than $12 l$, each such curve is contractible via the BCSP as a loop to any of the vertices of $k$. Let us, however, choose to contract it to the vertex with the smallest index. Here we use Lemma 1.3 to construct the required path homotopy between one side of $k$ and its two other sides. Next let us consider $k_{v_{0}, v_{1}, v_{2}, v_{3}}^{2} \in K_{2, l}$. Note that, as we know how to extend each $k_{v_{0}, \ldots, \hat{v}_{i}, \ldots, v_{3}}^{1}$, we, as the result of these extensions and natural identifications of the four 2-discs, have a map of the 2 -sphere into $M^{n}$ naturally assigned to $k_{v_{0}, v_{1}, v_{2}, v_{3}}^{2}$. Let us denote this (map of the) 2-sphere by $S_{0}^{2}$. We would like to construct a map of a 3 -disc that fills this 2 -sphere. It will be constructed as a 1-parameter family of 2 -spheres $S_{\tau}^{2}$ that begins with the original sphere obtained in the previous step $S_{0}^{2}$ and ends with a point. Here is how we will construct $S_{\tau}^{2}$. Let us begin by constructing a 1 -parameter family of graphs $k_{\tau}^{2}, \tau \in[0,2]$. We will let $k_{0}^{2}=k_{v_{0}, v_{1}, v_{2}, v_{3}}^{2}$. Next, by Lemma 1.3 there is a homotopy that moves edges $\left[v_{i}, v_{(i+1) \bmod 3]}\right], 0 \leq i \leq 2$, to $\left[v_{i}, v_{3}\right]+\left[v_{3}, v_{(i+1) \bmod 3}\right]$. This path homotopy passes through curves of length less than or equal to $4 l$. Let us denote the curves in these homotopies by $e_{\tau}^{i}$. So, we will continuously replace edges $e^{i}=\left[v_{i}, v_{(i+1) \bmod 3}\right]$ by the edges $e_{\tau}^{i}$, respectively, thus forming $k_{\tau}^{2}$. Let us now consider all the subcomplexes of $k_{\tau}^{2}$ that correspond to elements of $K_{1,4 l}$. By the previous step they can all be "filled" by 2-discs. Gluing these discs together results in a 2-sphere $S_{\tau}^{2}$. When $\tau=1$, this sphere will degenerate to (a map of the 2 -sphere into) a tree with root at $v_{3}$ and three edges connecting $v_{3}$ with $v_{0}, v_{1}, v_{2}$. This sphere fills a degenerate element of $K_{2,2 l}$ where all edges are mapped into this tree. This element can be contracted over itself to the constant map of the complete graph into $v_{3}$. Filling the resulting homotopy by spheres, we obtain a family of 2 -spheres $S_{\tau}^{2}, \tau \in[1,2]$ that connects $S_{1}^{2}$ and $S_{2}^{2}=\left\{v_{3}\right\}$. Thus, we obtain a 3 -disc that "fills" any $k_{v_{0}, \ldots, v_{3}}^{2} \in K_{2, l}$.

The following statement is an easy corollary to the above lemma.

Lemma 2.3. Let $f: K_{0} \longrightarrow M^{n}$ be a map from the 0-skeleton of a finite simplicial complex $K$ of dimension $r$ to a complete Riemannian manifold $M^{n}$. Let $v_{i}=f\left(\tilde{v}_{i}\right)$, where $\tilde{v}_{i} \in K_{0}$. Furthermore, suppose that $\sup d\left(v_{i}, v_{j}\right)=l$, where the supremum is taken over all pairs of vertices $\tilde{v}_{i}, \tilde{v}_{j} \in K_{0}$ that together bound a 1-simplex. Then if the infimum of the length of a shortest geodesic loop based at $v_{i}$ over all $v_{i}$ is 
greater than $3 \cdot 4^{r-1} l$, the map $f: K_{0} \longrightarrow M^{n}$ can be extended to a simplicial map $\tilde{f}: K \longrightarrow M^{n}$ in such a way that $\tilde{f}(K)$ is contained in the $6 \cdot 4^{r-2}$ l-neighborhood of $f\left(K_{0}\right)$.

Moreover, the restriction of $\tilde{f}$ to each 1-simplex of $K$ is a minimal geodesic connecting the images of the endpoints under $f$, and the restriction of $\tilde{f}$ to each simplex of $K$ is obtained from the restriction of $\tilde{f}$ to the 1-skeleton of this simplex and by application of the construction from the proof of Lemma 2.2 .

Proof. The extension procedure is inductive to the skeleta of $K$. Let us begin with the 1 -skeleton of $K$. Let $\left[\tilde{v}_{i}, \tilde{v}_{j}\right]$ be a 1-simplex of $K$. Then this simplex will be mapped to a shortest geodesic segment $\left[v_{i}, v_{j}\right]$ connecting $v_{i}$ and $v_{j}$. Now suppose we have extended $f$ to the $(i-1)$-skeleton of $K$. We will next extend it to the $i$-skeleton, which is accomplished by filling the image of its 1-skeleton as described in Lemma 2.2.

First enumerate all the vertices of the chosen triangulation of $K_{0}$ by increasing successive integers. Next apply Lemma 2.2 to previously constructed images of 1-skeleta of all $i$-dimensional simplices of $K_{0}$.

In order to do that, we need to renumber vertices of every $i$-dimensional simplex of $K_{0}$ by numbers $0,1, \ldots, i$. To do this we take the numbering of all of the vertices of $K_{0}$ and then renumber the vertices of every $i$-simplex by $\{0,1, \ldots, n\}$ in increasing order. Now apply Lemma 2.2. Notice that in the proof of Lemma 2.2, one uses only the fact that there are no short geodesic loops at the image points of $K_{0}$.

Note that after we finish the construction of the map, the image of each $r$ simplex will lie in the $6 \cdot 4^{r-2} l$-neighborhood of one of the $v_{i}$ 's. This also follows from Lemma 2.2

Lemma 2.4. Let $M^{n}$ be a complete Riemannian manifold, $p \in M^{n}$. Let $\varepsilon, \tau, \varrho$ be positive numbers, such that $\varrho<\frac{\varepsilon}{100^{n}}$. Define $\tilde{\varepsilon}$ by the equation

$$
6 \cdot 4^{n-1}\left(k(n-1) \tilde{\varepsilon}^{\frac{1}{n-1}}+2 \varrho+3 \tau\right)=\varepsilon,
$$

where $k(n-1)=27^{n-1} n$ !, and $\tau$ is sufficiently small for $\tilde{\varepsilon}$ to exist and to be positive. Suppose that given $t>\frac{\varepsilon}{2}$, there exists an $(n-1)$-dimensional submanifold $Z$ that lies within the @-tubular neighborhood of the geodesic sphere $\tilde{S}_{t}(p)$ centered at $p$ of radius $t$, such that

(1) $Z$ does not bound in $M^{n}-p$;

$(2) \operatorname{vol}(Z)<\tilde{\varepsilon}$. Then there exists a geodesic loop of length at most $\varepsilon$ based at a distance $t$ from the point $p$.

Proof. Let $X=L^{\infty}(Z)$. By Definition $0.4, Z$ isometrically embeds into $X$ and for every $\tau>0$ there exists a singular chain $c$ in the $(\operatorname{Fill} \operatorname{Rad}(Z)+\tau)$-neighborhood of $Z$ in $X$, such that $Z$ bounds $c$. Without loss of generality we can take $c$ to be an $n$-dimensional polyhedron (see Statement 1.2.C on page 10 in [G]). Also, recall that the Kuratowski embedding of $Z$ in $X$ is an isometry.

Assume that lengths of all nontrivial geodesic loops in $M^{n}$ based at the points of $\tilde{S}_{t}(p)$ are greater than

$$
(*) \varepsilon=6 \cdot 4^{n-1}\left(k(n-1) \tilde{\varepsilon}^{\frac{1}{n-1}}+2 \varrho+3 \tau\right)>6 \cdot 4^{n-1}\left(k(n-1) \operatorname{vol}_{n-1}(Z)^{\frac{1}{n-1}}+2 \varrho+3 \tau\right) .
$$

Gromov's Theorem 0.5 further implies that $\varepsilon>6 \cdot 4^{n-1}(\operatorname{Fill} \operatorname{Rad}(Z)+2 \varrho+3 \tau)$.

We will construct a finite $n$-chain in $M^{n}-p$ that has $Z$ as its boundary, thus obtaining a contradiction. This chain will be constructed in three steps. During 
the first step we will construct a simplicial map $f: Z \longrightarrow M^{n}-p$. During the second step we will extend the constructed map from $Z=\partial c$ into $M^{n}-p$ to a map $\tilde{f}: c \longrightarrow M^{n}-p$. Finally, we will show that the image of the fundamental class $[Z]$ under the homomorphism to $H_{n-1}\left(M^{n}-p\right)$ induced by the inclusion map and $\tilde{f}_{*}([Z])$ are equal, which would imply $Z$ bounds in $M^{n}-p$.

Step 1. Let $\operatorname{inj}(Z)=\inf _{x}\left\{\operatorname{inj} \operatorname{rad}_{x}\left(M^{n}\right), x \in Z\right\}$, where $\operatorname{inj} \operatorname{rad}_{x}\left(M^{n}\right)$ is the injectivity radius of the manifold $M^{n}$ at a point $x$. Triangulate $Z$ into simplices of size at most $\min \left\{\tau, \frac{\operatorname{inj}(Z)}{2}\right\}$. We will let the size of the simplices in a triangulation of $Z$ eventually go to 0 . We will begin by defining a map on the 0 -skeleton of $Z$. Each vertex $\tilde{v}_{i} \in Z$ is mapped to a closest point in $\tilde{S}_{t}(p)$, which is located within the distance $\frac{\varepsilon}{100^{n}}$. Next we will apply Lemma 2.3 in order to extend the map to the whole $Z$ with $l=2 \frac{\varepsilon}{100^{n}}+\tau$. Lemma 2.3 can be applied, because of our assumption about the length of a shortest geodesic loop at the distance $t$ from the point $p$.

Step 2. Triangulate $c$, a chain that fills $Z$ in the $\operatorname{Fill} \operatorname{Rad}(Z+\tau)$-neighborhood of $Z$ in the $L^{\infty}(Z)$, so that the diameter of each simplex is smaller than $\tau$. We will extend $f$ to the 0 -skeleton by mapping each vertex $\tilde{w}_{i}$ to a closest vertex $\tilde{v}_{i}$ in $Z$ and, subsequently, mapping it to a closest point $v_{i}$ in $\tilde{S}_{t}(p)$. Next, we apply Lemma 2.3 as in the previous step, with $l=2 \operatorname{Fill} \operatorname{Rad}(Z)+2 \varrho+3 \tau<\frac{\varepsilon}{3 \cdot 4^{n-1}}$.

Step 3. Finally we will construct an $n$-dimensional chain in $M^{n}-p$ with boundary components $Z$ and $\tilde{f}(Z)$.

The procedure will be an induction on the dimension of the skeleta that will go as follows: for each pair of simplices of dimension $n-1$, i.e. $\tilde{\sigma}_{j}^{n-1}$ of $Z$ and its image $\sigma_{j}^{n-1}=\tilde{f}\left(\tilde{\sigma}_{j}^{n-1}\right)$, we will construct a cell $\tau_{j}^{i}$ of the dimension $n$ that "connects" them.

This will be done by the filling technique similar to the one described in Lemma 2.2, but, while in Lemma 2.2. we needed to construct fillings of complete graphs, in this case we need to be able to construct fillings of objects that we will call racks. Here an $i$-rack will be (a map from) a simplicial complex that consists of two small simplices $\tilde{\sigma}_{j}^{i}$ and $\sigma_{j}^{i}$ and segments that connect the corresponding vertices of these simplices. As usual, "filling" should be understood as an extension of the map. In order to construct an $i$-skeleton of the complex, we will need to be able to construct fillings of $(i-1)$-racks with "short" edges.

We will begin with the 1-skeleton. The 1-skeleton will consist of minimal geodesic segments connecting a vertex of $Z$ with its image under the map $\tilde{f}$. Next we will construct the 2-cells. Consider the closed curves composed of a simplex $\left[\tilde{v}_{i_{1}}, \tilde{v}_{i_{2}}\right]$, its image $\left[v_{i_{1}}, v_{i_{2}}\right]$, and two minimal geodesic segments joining the corresponding vertices (note that this is a 1-rack with short edges). This closed curve can be contracted as a loop to either one of the vertices $v_{i_{1}}$ or $v_{i_{2}}$, because, by our assumption, there are no geodesic loops of sufficiently small length based at the points of $\tilde{S}_{t}(p)$. As in Lemma 2.2. we will contract the loop to the vertices with the smallest indices.

Let us assume that we are able to fill $(i-2)$-racks with edges of length not greater than $3 \cdot 4^{i-2} \frac{\varepsilon}{100^{n}}+\alpha$ for some sufficiently small $\alpha$ and have, thus, constructed the required $(i-1)$-skeleton.

To construct an $i$-skeleton of the chain, consider "prisms" $P_{j}^{i-1}$ that consist of the two simplices $\tilde{\sigma}_{j}^{i-1}$ of $Z$ and its image $\sigma_{j}^{i-1}$ together with the "walls", that is, 
cells of dimension $i-1$ that connect simplices of dimension $i-2$ in the boundaries of $\tilde{\sigma}_{j}^{i-1}$ and $\sigma_{j}^{i-1}$. We will construct a "filling" of this "prism", thus obtaining cells of dimension $i$. Informally speaking, the filling is obtained by first regarding the simplex $\tilde{\sigma}_{j}^{i-1}$ as a point $x$ and then applying Lemma 2.2 to the complete graph that has $x$ together with the vertices of $\sigma_{j}^{i-1}$ as its vertices.

More formally, let us use the induction assumption that we can continuously fill the $(i-2)$-racks with "short" edges, (i.e. of length at most $3 \cdot 4^{i-2} \frac{\varepsilon}{100^{n}}+\alpha$ for some small $\alpha$ ). Consider $P_{j}^{i-1}$, a prism that we are going to fill. Let $R_{j}^{i-1}$ be the corresponding rack. Let $q \in \tilde{\sigma}_{j}^{i-1}$ and consider a homotopy $h_{s}, s \in[0,1]$ that contracts $\partial \tilde{\sigma}_{j}^{i-1}$ to $q$ (see Figure $3(\mathrm{a})$ ). Without loss of generality, we can assume that the supremum of the length of the trajectories of the points of $\partial \tilde{\sigma}_{j}^{i-1}$ under this homotopy is at most some small $\tilde{\alpha}$ that will go to zero, as the diameter of simplices goes to zero. Let $\tilde{F}_{k}$ be an $(i-2)$-dimensional face of $\tilde{\sigma}_{j}^{i-1}$, and let $F_{k}$ be the corresponding face of $\sigma_{j}^{i-1}$. The homotopy $h_{s}$ generates a family of $(i-2)$ racks that correspond to $\tilde{F}_{k}$ in the following way. They are constructed by taking $\left(\tilde{F}_{k}\right)_{s}=h_{s}\left(\tilde{F}_{k}\right)$ and connecting each vertex $v$ of $F_{k}$ first with the corresponding vertex of $\tilde{v}$ of $\tilde{F}_{k}$ and then with $h_{s}(\tilde{v})$ by a path of length at most $\tilde{\alpha}$, which is simply a part of the trajectory of the vertex $\tilde{v}$. At each time $s$ we are using an induction assumption to fill the corresponding rack. At the time $s=1$, the image of all of the faces of $\tilde{\sigma}_{j}^{i-1}$ is the point $q$ and so, the last step is to fill a simplicial complex that consists of the simplex $\sigma_{j}^{i-1}$ that is connected with the point $q$ by short segments. This step is illustrated by Figure 3, where the above row (see Figure 3 (b)) depicts a 1-parameter family of racks that correspond to $\left(\tilde{\sigma}_{j}^{i-1}\right)_{s}=h_{s}\left(\tilde{\sigma}_{j}^{i-1}\right)$, beginning with $R_{j}^{i-1}$ and ending with the one that corresponds to the point $q$, and the bottom row consists of the corresponding prisms obtained from the racks by continuously filling in the missing faces. Note that the original map is unaffected by this process.

We can now apply Lemma 2.2 to the 1 -skeleton $k_{i, j}$ of this simplicial complex to obtain a filling $\phi_{i-1}\left(k_{i, j}\right) \in C\left(\sigma^{i}, M^{n}\right)$. Note that condition (2) of Definition 2.1 together with Lemma 2.2 guarantees the coherence of the filling in the following sense. Recall that the simplex $\sigma_{j}^{i-1}$ is also constructed by applying Lemma 2.2 to the 1-skeleton of this simplex (see Step 2 above), thus the restriction of $\phi_{i-1}$ to an $(i-1)$-face of $\phi_{i-1}\left(k_{i, j}\right)$ coincides with the filling of the 1-skeleton of the face, and, in particular, the restriction of the filling to the face obtained from $\phi_{i-1}\left(k_{i, j}\right)$ by removing $x$ is the simplex $\sigma_{j}^{i-1}$.

Thus, the existence of a procedure of a continuous filling of $(i-2)$-racks implies the existence of filling of $(i-1)$-racks, which implies the existence of filling of $(i-1)$ prisms, which generates the $i$-skeleton of the complex that we are constructing. (This argument is similar to the Remark on pg. 504 in [R3], as well as an argument in [NR5.)

Proof of Theorem 0.1 . Let $p \in M^{n}$ and $\varepsilon>0$ be given. Let $\tilde{\varepsilon}=\left(\frac{\frac{\varepsilon}{6 \cdot 4^{n-1}}-\sigma}{k(n-1)}\right)^{n-1}$, where $k(n-1)=27^{n-1} n$ ! and for some small $\sigma$ that we, eventually, will let go to zero.

Then by Lemma 1.1 there exists a set $A(\tilde{\varepsilon})$ satisfying the following condition: At each $t^{*}$ in the complement of $A(\tilde{\varepsilon})$ in $(0, \infty)$, there exists a geodesic sphere $\tilde{S}_{t^{*}}(p)$ 


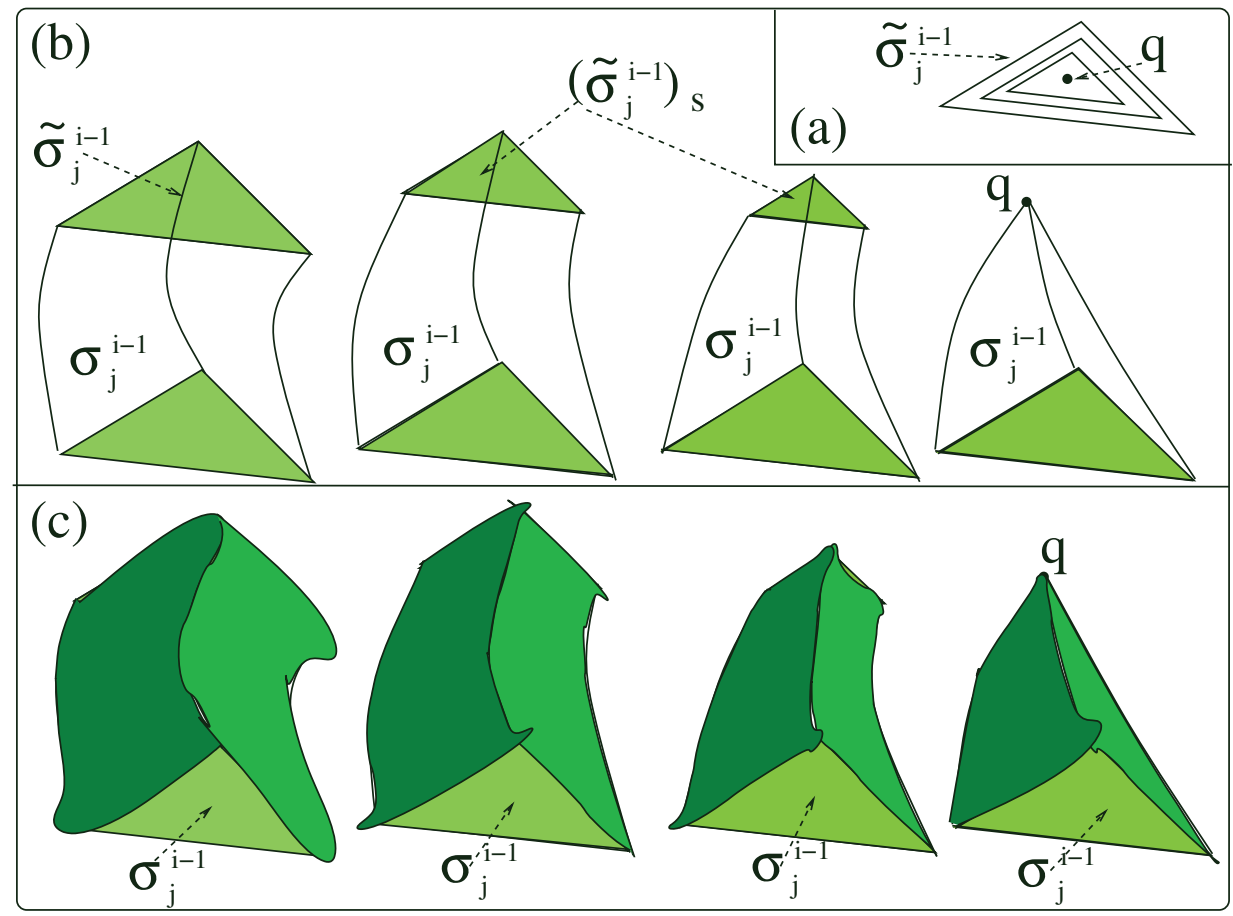

Figure 3. A filling of an $i$-rack.

of radius $t^{*}$ centered at the point $p$ such that for any $\delta>0$ there exists a smooth submanifold $Z_{\tilde{\varepsilon}}^{\delta}$ that has $\operatorname{vol}_{n-1}\left(Z_{\tilde{\varepsilon}}^{\delta}\right)<\tilde{\varepsilon}$ and that is within distance $\delta$ from the sphere. Moreover, $Z_{\tilde{\varepsilon}}^{\delta}$ does not bound in $M^{n}-p$.

Take $t^{*}>T=4^{n} k(n-1) \tilde{\varepsilon}^{\frac{1}{n-1}}=\frac{2 \varepsilon}{3}-4 \sigma>\frac{\varepsilon}{2}$.

Let $i_{S}=\inf _{q \in \tilde{S}_{t^{*}(p)}} \operatorname{inj}_{q} M^{n}$, where $\operatorname{inj}_{q} M^{n}$ is the injectivity radius of $M^{n}$ at $q$. We will consider $\delta<\frac{i_{S}}{100^{n}}$, which will eventually approach 0 .

Now let us apply Lemma 2.4 in which we take $Z=Z_{\tilde{\varepsilon}}^{\delta}$ and $\varrho=\delta$ that will eventually go to 0 .

Next we will present a proof of Theorem 0.3 .

Proof of Theorem 0.3 . Let $\varepsilon>0$ be given. Without any loss of generality we can assume that $\varepsilon \leq 1$. Let $\tilde{\varepsilon}=\left(\frac{\varepsilon}{12 \cdot 4^{n-2} 27^{n-1} n !}\right)^{n-1}$. First we will show that there exists $T>0$, such that for all $t>T$ there exists a $\tilde{t}$, such that $|\tilde{t}-t|<\frac{\tilde{\varepsilon}}{100^{n}}$ and $\operatorname{vol}_{n-1}\left(\tilde{S}_{\tilde{t}}(p)\right)<\frac{\tilde{\varepsilon}}{16}$. This is easily seen from the fact that the maximal number of the disjoint intervals of length $\frac{2 \tilde{\varepsilon}}{100^{n}}$, such that the measure of every geodesic sphere with the radius in one of these intervals is greater than $\frac{\tilde{\varepsilon}}{16}$ is finite. Indeed, let $\tilde{T}=\sup \left\{\left(a_{\alpha}, b_{\alpha}\right), \alpha \in \Lambda\right\}$, where $\left\{\left(a_{\alpha}, b_{\alpha}\right), \alpha \in \Lambda\right\}$ is the set of all intervals of length $\frac{2 \tilde{\varepsilon}}{100^{n}}$, such that the measure of every geodesic sphere with the radius in $\left(a_{\alpha}, b_{\alpha}\right)$ is greater than $\frac{\tilde{\varepsilon}}{16}$, and take $T=\max \left\{\frac{\varepsilon}{2}, \tilde{T}\right\}$.

Now suppose $t>T$ is given. Then there exists $\tilde{t}$ satisfying the above property. Moreover, by Lemma 1.1 for every $\delta$ that is small enough there exists a submanifold 
$Z_{\tilde{\varepsilon}}^{\delta}$ such that $\operatorname{vol}_{n-1}\left(Z_{\tilde{\varepsilon}}^{\delta}\right)<\tilde{\varepsilon}$ within distance $\delta$ from $\tilde{S}_{\tilde{t}}(p)$ that does not bound in $M^{n}-p$. Now, let us apply Lemma 2.4

\section{ACKNOWLEDGMENTS}

This paper was partially written during the author's visit to Tel-Aviv University in July 2008 and to the Max-Planck-Institüt fur Matematics, Bonn, in the summer of 2009. The author would like to thank Tel-Aviv University and the Max-PlanckInstitüt for their kind hospitality. The author gratefully acknowledges a partial support of Natural Sciences and Engineering Research Council (NSERC) University Faculty Award and Research Grant, and a partial support of her NSF Research Grant DMS-0604113 during her work on the present paper. Finally, the author would like to thank D. Burago for the suggestion to look for upper bounds for the lengths of stationary objects on noncompact manifolds of finite volume.

\section{REFERENCES}

[B] V. Bangert, Closed geodesics on complete surfaces, Math. Ann., 251 (1980), 83-96. MR583827 (81k:58027)

[Bl] F. Balacheff, Sur des problemes de la géométrie systolique, Séminaire de Théorie Spectrale et Géométrie. Vol. 22. Année 2003-2004, 71-82, Sémin. Théor. Spectr. Géom., 22, Univ. Grenoble I, Saint-Martin-d'Hères, 2004. MR2136136 (2006a:53038)

[BZ] Ju. Burago, V. Zalgaller, Geometric Inequalities, Berlin: Springer-Verlag, 1988. MR936419 (89b:52020)

[C] C. B. Croke, Area and the length of the shortest closed geodesic, J. Diff. Geom., 27 (1988), 1-21. MR918453 (89a:53050)

[CK] C. B. Croke, M. Katz, Universal volume bounds in Riemannian manifolds, Surveys in Differential Geometry. Vol. VIII (Boston, MA 2002), 109-137, Surv. Diff. Geom. VIII, Int. Press. Somerville, MA, 2003. MR2039987 (2005d:53061)

[Ga] M. Gaffney, The conservation property of the heat equation on Riemannian manifolds, Comm. Pure Appl. Math., 12 (1259), 1-11. MR0102097(21:892)

[G] M. Gromov, Filling Riemannian manifolds, J. Diff. Geom., 18 (1983), 1-147. MR697984 (85h:53029)

[Gt1] L. Guth, Volume of balls in large Riemannian manifolds, Ann. Math. (2) 173(2011), no. 1, 57-76. MR 2753599

[M] M. Maeda, The length of a closed geodesic on a compact surface, Kyushu J. Math., 48 (1994), no. 1, 9-18. MR1269063 (95b:58035)

[NR1] A. Nabutovsky, R. Rotman, The length of the shortest closed geodesic on a 2-dimensional sphere, IMRN 2002, no. 23, 1211-1222. MR1903953 (2003d:53062)

[NR2] A. Nabutovsky, R. Rotman, Volume, diameter and the minimal mass of a stationary 1cycle, Geom. Funct. Anal., 14 (2004), no. 4, 748-790. MR2084979 (2005g:53069)

[NR3] A. Nabutovsky, R. Rotman, Curvature-free upper bounds for the smallest area of a minimal surface, Geom. Funct. Anal., 16 (2006), no. 2, 453-475. MR2231470 (2007d:53062)

[NR4] A. Nabutovsky, R. Rotman, The minimal length of a non-trivial net on a closed Riemannian manifold with a non-trivial second homology group, Geom. Dedicata, 113 (2005), no. 1, 243-254. MR2171308 (2006g:53048)

[NR5] A. Nabutovsky, R. Rotman, The length of the second shortest geodesic, Comment. Math. Helv., 84 (2009), 747-755. MR2534478(2011b:53085)

[R1] R. Rotman, The length of a shortest closed geodesic and the area of a 2-dimensional sphere, Proc. Amer. Math. Soc., 134 (2006), no. 10, 3041-3047. MR2231630 (2007f:53039)

[R2] R. Rotman, The length of a shortest geodesic net on a closed Riemannian manifold, Topology, 46:4 (2007), 343-356. MR2321036 (2008m:58026)

[R3] R. Rotman, The length of a shortest geodesic loop at at point, J. Diff. Geom., $78: 3$ (2008), 497-519. MR2396252(2008m:53092)

[R4] R. Rotman, Flowers on Riemannian manifolds, preprint, MPIM2006-104, available at www.mpim-bonn.mpg.de/preprints/retrieve. 
[S1] S. Sabourau, Filling radius and short closed geodesics of the 2-sphere, Bull. Soc. Math. France, 132 (2004), no. 1, 105-136. MR2075918 (2005g:53065)

[S2] S. Sabourau, Global and local volume bounds and the shortest geodesic loops, Commun. Anal. Geom., 12 (2004), 1039-1053. MR2103310(2005h:53060)

[T] G. Thorbergsson, Closed geodesics on non-compact Riemannian manifolds, Math. Z., 159 (1978), 249-258. MR0493872 (58:12833)

[W] S. Wenger, A short proof of Gromov's filling inequality, Proc. Amer. Math. Soc., 136 (2008), 2937-2941. MR2399061 (2009a:53072)

Department of Mathematics, Pennsylvania State University, University Park, PennSYLVANIA 16802

E-mail address: rotman@math.psu.edu

Current address: Department of Mathematics, University of Toronto, 40 St. George Street, Toronto, Ontario, Canada M5S 2E4

E-mail address: rina@math.toronto.edu 